\title{
ПРАКТИЧЕСКИЕ РЕКОМЕНДАЦИИ ПО ЛЕЧЕНИЮ ПЕРВИЧНЫХ ЗЛОКАЧЕСТВЕННЫХ ОПУХОЛЕЙ КОСТЕЙ (ОСТЕОСАРКОМЫ, САРКОМЫ ЮИНГА)
}

\section{РЕКОМЕНДАЦИИ ВОСТОЧНО-ЕВРОПЕЙСКОЙ ГРУППЫ ПО ИЗУЧЕНИЮ САРКОМ}

Коллектив авторов: Феденко А. А., Бохян А. Ю., Горбунова В. А., Махсон А. Н., Тепляков В. В. DOI: $10.18027 / 2224-5057-2020-10-3 s 2-17$

Ключевые слова: саркомы костей, химиотерапия, остеосаркома, саркома Юинга, ПНЭО

Саркомы - редкая группа опухолей. В России ежегодно регистрируется около 10000 новых случаев, что составляет 1 \% всех злокачественных новообразований. В 50\% случаев первичные злокачественные опухоли костей представлены остеосаркомой, далее следуют саркомы семейства Юинга. Остальные гистологические формы встречаются редко. Примерно $50 \%$ костных сарком диагностируется в возрасте до 35 лет и гораздо чаще встречаются у детей и молодых людей, во втором десятилетии жизни, когда их частота равна 3 на 100000 населения. У лиц старше 30 лет заболеваемость составляет 0,2 на 100000 населения. Остеосаркома чаще всего возникает в период быстрого роста костей, и поэтому риск этой опухоли несколько больше у высоких подростков, особенно у мальчиков. Кроме того, повышенный риск остеосаркомы наблюдается при некоторых генетических аномалиях: у детей с наследственной ретинобластомой, синдромом Ли-Фраумени. Около $10 \%$ заболевших пожилые люди старше 60 лет; считается, что у них развитие опухоли может быть спровоцировано другими костными заболеваниями, возникшими к этому возрасту, или связано с перенесённым облучением. Мужчины болеют несколько чаще женщин. В России на протяжении многих лет заболеваемость сохраняется на уровне 2,13 и 1,83 случая на 100000 населения среди мужчин и женщин соответственно.

Цитирование: Феденко А.А., Бохян А.Ю., Горбунова В.А., Махсон А.Н., Тепляков В.В. Практические рекомендации по лечению первичных злокачественных опухолей костей (остеосаркомы, саркомы Юинга). Злокачественные опухоли: Практические рекомендации RUSSCO \#3s2, 2020 (том 10).17 


\section{Остеосаркомы}

\section{1. КЛАССИФИКАЦИЯ И ОПРЕДЕЛЕНИЕ СТАДИИ}

Стадирование злокачественных опухолей костей должно проводиться по системе TNM (7-е издание, 2011 г.) (табл. 1).

Таблица 1. Система стадирования злокачественных опухолей костей

\begin{tabular}{|l|l|l|l|l|l|}
\hline Стадия & T & N & M & $\begin{array}{l}\text { Степень злокаче- } \\
\text { ственности (G) }\end{array}$ & Локализация опухоли \\
\hline IA & T1 & N0 & M0 & Низкая & $\leq 8$ см \\
\hline IB & T2 & N0 & M0 & Низкая & $>8$ см \\
\hline IIA & T1 & N0 & M0 & Высокая & $\leq 8$ см \\
\hline IIB & T2 & N0 & M0 & Высокая & > 8 см \\
\hline III & T3 & N0 & M0 & Любая & $\begin{array}{l}\text { Опухоль распространилась } \\
\text { на другие зоны }\end{array}$ \\
\hline IVA & Любая T & N0 & М1а (только лёгкие) & Любая & Любая \\
\hline IVB & Любая T & N1 & Любая M & Любая & Любая \\
\cline { 2 - 6 } & Любая T & Любая N & М1ь & Любая & Любая \\
\hline
\end{tabular}

\section{2. ДИАГНОСТИКА}

\section{Общие принципы диагностики}

Основными клиническими признаками опухолей костей являются припухлость, боль, нарушение функции конечности. Для костных сарком характерны упорные прогрессирующие, усиливающиеся по ночам боли, которые плохо купируются анальгетиками. Участки структурной перестройки кости становятся видимыми только при достижении определённых размеров, которые зависят от расположения очага, объёма поражённой части кости, архитектоники губчатого вещества, массива окружающих мягких тканей и т. д. Во избежание диагностических ошибок необходимо применять радионуклидную диагностику и МРТ (рис. 1). В план обследования больных саркомами костей должны входить:

- анализ анамнестических данных и жалоб, клинический осмотр;

- лабораторные методы обследования: полный общий анализ крови, биохимический анализ крови (мочевина, креатинин, электролиты, оценка функции печени), коагулограмма, ЭКГ, ЭхоКГ;

- R-графия поражённого сегмента скелета в 2-х стандартных проекциях;

- MPT + КТ поражённого костного сегмента; 
- $\mathrm{KT}$ (предпочтительно) или R-графия органов грудной клетки в прямой и боковой проекциях;

- Узи брюшной полости, забрюшинного пространства, периферических лимфоузлов, поражённого сегмента;

- сцинтиграфия костей скелета;

- биопсия + гистологическое исследование биоптата опухоли.

Выполнение ПЭТ с целью первичного стадирования не рекомендуется.

\section{1. Биопсия}

Диагноз устанавливается на основании гистологического исследования биоптата опухоли. Вне зависимости от способа биопсии (трепанобиопсия или открытая биопсия) забор материала должен производиться наименее травматично для пациента. Зона биопсии должна быть включена в блок удаляемых во время хирургического этапа тканей. Поскольку нарушение целостности кости может спровоцировать патологический перелом, биопсию внутрикостного фрагмента опухоли необходимо проводить только в тех случаях, когда внекостный компонент опухоли отсутствует или слабо выражен. Выполнение толстоигольной биопсии оправдано малым количеством осложнений и возможностью навигации при помощи КТ и УЗИ, однако в $20 \%$ случаев может потребоваться повторное выполнение процедуры в связи с малым количеством получаемого материала. Проведение биопсии опухоли открытым доступом допустимо при анатомически трудной локализации очага опухоли, или, как второй этап, при неинформативной трепанобиопсии. При выполнении биопсии опухоли основополагающим топографическим критерием её проведения является стандартный оперативный доступ предстоящего оперативного лечения. Зона забора биопсийного материала всегда определяется онкоортопедом, выполняющим хирургический этап лечения.

\section{2. Морфологическое исследование}

Морфологическая диагностика представляет большую сложность. Цитологическое исследование для постановки диагноза не используется. Отсутствие стандартных наборов ИГХ реакций и цитогенетических исследований в значительной степени повышают роль патоморфолога, от квалификации и опыта которого зависит постановка правильного гистологического диагноза. В настоящее время выделяется более 5 форм остеосарком с разными степенями дифференцировки.

Цитогенетическое исследование: хромосомные изменения описаны при многих саркомах. В настоящее время их идентификация используется только для более тщательной диагностики того или иного гистологического подтипа, в частности высока их роль в диагностике ПнЭо/сарком семейства Юинга.

К первичным злокачественным опухолям костей относится хондросаркома, основным методом лечения которой является хирургический, за исключением случаев дедифференцировки в остеосаркому или злокачественную фиброзную 
гистиоцитому (комбинированное лечение по стандартам остеосаркомы) или мезенхимальный гистологический подтип (лечение по стандартам сарком семейства Юинга).

\section{3. ЛЕЧЕНИЕ}

\section{Основные принципы первичного лечения локализованных остеосарком}

Лечение больных с локализованной формой остеосаркомы является комплексным и включает предоперационную XT, операцию, послеоперационную химиотерапию. Хирургическое лечение в самостоятельном варианте нецелесообразно, т. к. у 80-90\% больных в течение 1,5 лет после операции диагностируются метастазы в лёгких и локальные рецидивы. В связи с этим лечение первичных больных должно проводиться только в специализированных центрах и требует комплексного подхода с привлечением ряда специалистов: морфолога, лучевого диагноста, хирурга, радиолога, химиотерапевта. лТ терапия используется только в сочетании с ХТ. Методика облучения, доза и источник зависят от гистологического подтипа опухоли, возраста больного, локализации и размеров опухоли, характера планируемой или проведённой операции, её радикальности. Применение ХТ в сочетании с оперативным пособием позволяет улучшить выживаемость больных как с локализованным, так и метастатическим процессом.

Современным стандартом хирургического лечения следует считать органосохраняющие вмешательства (эндопротезирование длинных трубчатых костей, эндопротезирование мелких суставов, резекции костей таза с этапами восстановления целостности тазового кольца, вертебрэктомии с восстановлением целостности позвоночного столба, резекции грудной клетки с восстановлением механической и дыхательной функции и т. д.). С учётом преимущественно молодого возраста пациентов особое внимание следует обратить на характер операций. В настоящее время калечащие операции допустимы не более, чем в $10 \%$ случаев и выполняются при отсутствии эффекта консервативного лечения. Хирургическое лечение должно выполняться только в специализированных учреждениях онкологического профиля с применением инновационных высокотехнологичных методов, направленных на максимальное сохранение поражённого отдела скелета. Реабилитация пациентов является важным этапом лечения, позволяющим улучшить качество жизни как после органосохраняющих, так и после калечащих операции, ускорить процесс социализации пациента (рис. 2).

\section{1. Хирургическое лечение}

Органосохраняющие операции являются стандартом лечения пациентов с диагнозом саркома кости. Главным условием операбельности пациентов 
с диагнозом «саркома кости» является возможность радикального и абластичного удаления опухоли, что гарантирует отсутствие местного рецидива.

\section{Виды радикального оперативного лечения}

Ампутация или экзартикуляция конечности выполняются в следующих случаях:

- обширное первично-множественное распространение опухоли;

- вовлечение в опухолевый процесс магистрального сосудисто-нервного пучка, технически исключающее проведение реконструктивно-пластического этапа операции;

- прогрессирование на фоне предоперационной ХT;

- отказ пациента от органосохраняющей операции;

- жизненные показания к экстренному хирургическому вмешательству в связи с распадом опухоли и/или кровотечением.

В остальных случаях в первую очередь следует рассматривать возможность выполнения органосохраняющих операций. Размер первичного очага опухоли, степень распространения внекостного компонента не могут использоваться как самостоятельные независимые критерии при решении вопроса о выполнении калечащей операции. Костные резекции выполняются при наличии минимального необходимого отступа от предполагаемого края опухоли не менее 3-5 см (при отсутствии распространения опухоли в костномозговом канале оставшейся части кости).

\section{Особенности хирургической тактики в зависимости ог формы остеосаркомы}

- Параостальная (юкстакортикальная) остеосаркома: только радикальное хирургическое лечение (вне зависимости от степени распространения опухоли).

- Периостальная остеосаркома: радикальное хирургическое лечения. В зависимости от размера первичного очага и степени распространения опухоли на консилиуме в составе хирурга онкоортопеда и химиотерапевта принимается решение о целесообразности консервативного лечения. XT в ряде случаев может уменьшить размеры первичного очага и метастазов опухоли, способствовать её отграничению псевдокапсулой и перевести в резектабельное состояние.

- Остеосаркома высокой степени злокачественности: комбинированное лечение, включающее неоадьювантную ХT, радикальное хирургическое лечение, адъювантную ХТ.

\section{Эндопротезирование}

В современной онкоортопедии среди различных способов восстановления дефекта и функции конечности после удаления опухолевого очага ведущее место занимают высокотехнологичные операции с использованием эндопротезов. Использование модульных систем эндопротезирования является предпочтитель- 
ным способом реконструкции костных дефектов при саркомах кости. Выделяют следующие виды реконструктивных операций в объёме эндопротезирования:

- стандартные первичные операции у взрослых;

- стандартные первичные операции у детей и подростков;

- эндопротезирование редких анатомических зон;

- ревизионное эндопротезирование.

Требования, предъявляемые к конструкции эндопротеза при первичном эндопротезировании у взрослых:

- модульность конструкции эндопротеза;

- максимальная надёжность и ремонтопригодность эндопротеза;

- универсальность компонентов эндопротеза;

- максимальный срок службы;

- стабильная фиксация эндопротеза;

- хороший функциональный результат;

- короткий срок изготовления эндопротеза.

Особенности эндопротеза при первичном эндопротезировании у детей и подростков:

- наличие бесцементного способа фиксации эндопротеза;

- раздвижной механизм эндопротеза (инвазивный и неинвазивный).

Рост количества случаев первичного эндопротезирования и успехи XT костных сарком приводят к ежегодному увеличению количества пациентов, нуждающихся в повторных ортопедических операциях в связи с нестабильностью или поломкой эндопротеза. Проведение ревизионных операций вне зависимости от их причины требует подготовленной команды онкоортопедов и соответствующего оборудования. Ревизионный эндопротез изготавливается индивидуально, что позволяет, не удаляя весь эндопротез, заменить его часть. Применение модульной системы эндопротезирования упрощает выполнение последующих ревизионных операций и увеличивает биологический резерв кости.

\section{2. Химиотерапия}

ХT как часть комплексного лечения значительно улучшает 5-летнюю выживаемость у больных с локализованным процессом (с $20 \%$ до $60 \%$ ). Преимущества предоперационной ХТ заключаются в возможности оценки in vivo активности химиотерапевтических препаратов и облегчении выполнения операции. Обычно проводится 3-4 цикла предоперационной ХT.

Целесообразность послеоперационной ХТ определяется степенью лекарственного патоморфоза. Степень некроза опухоли после предоперационной ХT - надёжный независимый прогностический фактор. При выраженном ответе опухоли на проведённое лечение (некроз опухоли $\geq 90 \%$ ) имеется большая вероятность увеличения безрецидивной и общей выживаемости. При отсутствии эффекта предоперационной ХТ послеоперационная ХТ не 
приводит к улучшению выживаемости. При III - IV степени повреждения опухоли (более $90 \%$ опухолевой ткани некротизировано) послеоперационно проводится 4 курса ХТ с использованием тех же цитостатиков; при меньшей степени некроза длительность лечения составляет около 12 мес. с использованием альтернирующих схем, включающих 5-6 цитостатиков.

При лечении локализованного опухолевого процесса используется интенсивная XT на основе доксорубицина и цисплатина. При низкой клинической и R-логической эффективности 2 циклов схема лечения меняется на 2 цикла высокодозной ХТ ифосфамидом. Многие центры включают в схему ифосфамид или высокие дозы метотрексата (+кальция фолинат); при крайне низкой степени лечебного патоморфоза могут также использоваться комбинации гемцитабина и доцетаксела, а также дакарбазина и доксорубицина. Продолжительность ХТ варьирует от 7 до 12 мес. Обязательным условием проведения ХT является строгое соблюдение методики введения высоких доз метотрексата, ифосфамида, цисплатина. Использование меторексата оправдано у пациентов молодого возраста, эффективность у больных старше 25-30 лет сомнительна.

Изредка диссеминированные формы остеосаркомы ошибочно принимают за злокачественную гигантоклеточную опухоль кости, которая нечувствительна к цитотоксическим препаратам, но обладает высокой чувствительностью к деносумабу, который назначается по 120 мг п/к 1 раз в 4 нед. (в первый мес.- в 1-й, 8-й, 15-й и 28-й дни). Рекомендуемые режимы ХТ остеосарком представлены в табл. 2.

таблица 2. Рекомендуемые режимы химиотерапии остеосарком

\begin{tabular}{|c|c|}
\hline AP & $\begin{array}{l}\text { Доксорубицин } 90 \text { мг/м² в/в 96-часовая инфузия+ цисплатин } 120 \text { мг/м² в/в или в/а инфузия } \\
\text { в 1-й день+ Г-КСФ (филграстим } 5 \text { мкг/кг п/к в 5-15-й дни) каждые } 4 \text { нед., } 4 \text { цикла }\end{array}$ \\
\hline$H D^{1} I$ & $\begin{array}{l}\text { Ифосфамид } 2000 \text { мг/м² в/в в 1-7-й дни (+ месна) + Г-КСФ (филграстим } 5 \text { мкг/кг п/к в 8-16-й дни), } \\
2 \text { цикла }\end{array}$ \\
\hline \multicolumn{2}{|c|}{ Альтернативный вариант после операции } \\
\hline \multicolumn{2}{|c|}{ Некроз опухоли более 90\% } \\
\hline $\begin{array}{l}\mathrm{HD} \text { Al } \\
(75 / 10)\end{array}$ & $\begin{array}{l}\text { Доксорубицин } 25 \text { мг/м²/сут. в/в в 1-3-й дни (72-часовая непрерывная инфузия) + ифосфамид } \\
2500 \text { мг/м² (+ месна) в/в в 1-4-й дни+ Г-КСФ (филграстим } 5 \text { мкг/кг п/к в 5-15-й дни), } 4 \text { цикла }\end{array}$ \\
\hline \multicolumn{2}{|c|}{ Некроз опухоли 50-90\% } \\
\hline HD I & $\begin{array}{l}\text { Ифосфамид } 2000 \text { мг/м² в/в (+ месна) в 1-7-й дни+ Г-КСФ (филграстим } 5 \text { мкг/кг п/к в 8-16-й дни), } \\
\text { каждые } 3 \text { нед., } 2 \text { цикла } \\
\text { Через } 3 \text { нед.: }\end{array}$ \\
\hline HD Mtx & $\begin{array}{l}\text { Метотрексат } 12 \text { г/м² в/в (+ кальция фолинат), каждые } 2 \text { нед., } 4 \text { введения } \\
\text { Через } 3 \text { нед.: }\end{array}$ \\
\hline $\begin{array}{l}\mathrm{HD} \text { Al } \\
(75 / 10)\end{array}$ & $\begin{array}{l}\text { Доксорубицин } 25 \text { мг/м²/сут. в/в в 1-3 дни (72-часовая непрерывная инфузия) + ифосфамид } \\
2500 \text { мг/м² (+ месна) в/в в 1-4-й дни+ Г-КСФ (филграстим } 5 \text { мкг/кг п/к в 5-15-й дни), } 2 \text { цикла } \\
\text { Все три схемы повторяются } 3 \text { раза }\end{array}$ \\
\hline \multicolumn{2}{|c|}{$\begin{array}{l}\text { Некроз опухоли менее } 50 \% \text { : } \\
\text { применяются те же схемы трижды, но с заменой режима HD Al на режим GemTax }\end{array}$} \\
\hline GemTax & $\begin{array}{l}\text { Гемцитабин } 900 \text { мг/м² в/в в 1-й и 8-й дни (90-минутная инфузия) + доцетаксел } \\
100 \text { мг/м² в/в в 8-й день+ Г-КСФ (филграстим } 5 \text { мкг/кг п/к в 9-19-й дни), } 2 \text { цикла }\end{array}$ \\
\hline
\end{tabular}

1 HD-высокие дозы 


\section{3. Лечение метастатического процесса и рецидивов}

При лечении метастатических форм используется комбинированная XT в сочетании с возможным оперативным лечением. Выбор XТ II линии зависит от первичного лечения. Чаще всего используются высокие дозы метотрексата, ифосфамид. Использование этопозида не оправдано, так как его эффективность в лечении остеосарком не доказана. При появлении метастазов более, чем через 6 мес. после комплексного лечения возможно повторение ранее использованного режима ХТ. При более раннем прогрессировании следует применять другие комбинации цитостатиков.

При метастатическом поражении лёгких следует обсудить возможность оперативного вмешательства в объёме метастазэктомии, повторной торакотомии в комплексе с ХТ. При метастазах в лёгких их полное хирургическое удаление может обеспечить достижение $40 \%$-ной 5-летней выживаемости.

Используются те же режимы ХТ в такой же последовательности, как и для лечения первичной опухоли. При наличии только лёгочных метастазов у не леченных (первичных) больных ХТ должна проводиться не с паллиативной, а лечебной целью, т. е. с соблюдением стандартных для лечения данной патологии режимов (без редукции доз и увеличения интервалов между курсами), т. к. в $20 \%$ можно достичь 5-летней выживаемости без признаков заболевания. Кроме стандартных режимов, в качестве III и последующих линий терапии возможно применение сорафениба по 400 мг × 2 раза/сут. внутрь ежедневно.

\section{4. НАБЛЮДЕНИЕ}

Больные остеосаркомой, закончившие этап комбинированного лечения, должны наблюдаться с периодичностью 1 раз в 3 мес. в течение первых 2 лет, далее - 1 раз в 6 мес. до общей продолжительности 5 лет Обязательными процедурами являются выяснение жалоб, осмотр, КТ грудной клетки, УзИ области послеоперационного рубца, R-графия зоны костного поражения.

Рекомендуемый алгоритм лечебно-диагностических мероприятий при остеогенной саркоме представлен на рис. 1 и 2.

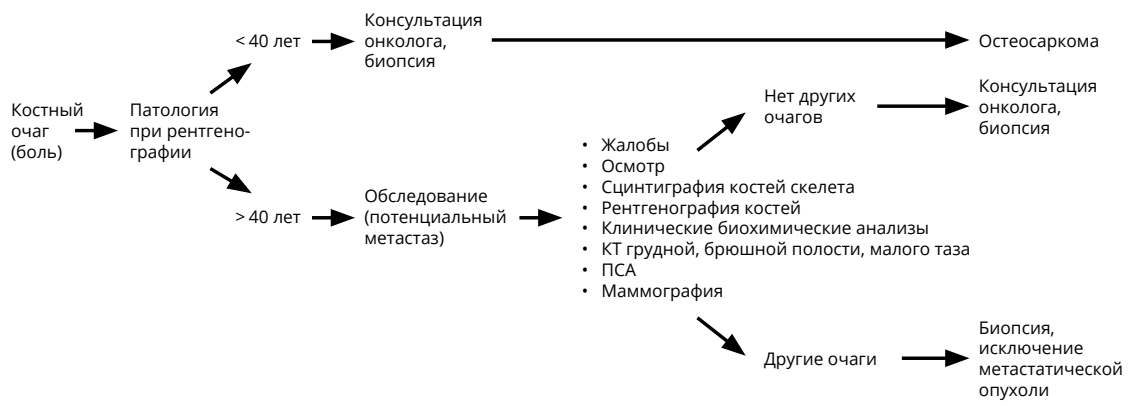

Рисунок 1. Рекомендуемый диагностический алгоритм при остеосаркоме. 
ОБСЛЕДОВАНИЕ

- Осмотр

- МРТ или КТ первичного очага

- КТ грудной клетки

- Сцинтиграфия костей скелета

- лдГ

- ЩФ
ПЕРВИЧНОЕ ЛЕЧЕНИЕ

АДЪЮВАНТНОЕ ЛЕЧЕНИЕ

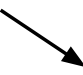

Высокой

степени \локаче- Лечение

ственности

Химиотерапия: в/в или в/а, включает не менее двух цитостатиков (доксорубицин, цисплатин, ифосфамид, высокие дозы метотрексата), Г-КСФ.

Рисунок 2. Рекомендуемый лечебно-диагностический алгоритм при остеосаркоме.

\section{Саркома Юинга}

\section{1. КЛАССИФИКАЦИЯ И ОПРЕДЕЛЕНИЕ СТАДИИ}

В 2020 г. опубликовано 5-е издание «Классификации ВОЗ опухолей мягких тканей и костей», в которой выделена новая отдельная группа опухолей костей и мягких тканей - недифференцированные мелкокруглоклеточные саркомы костей и мягких тканей (синоним: злокачественные мелкокруглоклеточные опухоли семейства саркомы Юинга).

В данную группу недифференцированных мелкоклеточных сарком костей и мягких тканей включены 4 нозологические группы:

9364/3 Саркома Юинга

9366/3 Круглоклеточная саркома с транслокацией химерного гена EWSR7non-ETS

9367/3 Саркома с перестройкой гена CIC (CIC-DUX4)

9368/3 Саркома с перестройкой гена BCOR.

Стадирование не отличается от общепринятой классификации для первичных опухолей костей, детально описанной в разделе остеосарком. При внекостной форме стадирование проводится по системе для опухолей мягких тканей. 


\section{2. ДИАГНОСТИКА}

С учётом высокой частоты метастазирования в кости и костный мозг в стандартный алгоритм диагностических процедур для первичных опухолей костей дополнительно включены сцинтиграфия костей скелета и трепанобиопсия костного мозга (рис. 3).

\section{3. ЛЕЧЕНИЕ}

Саркомы семейства Юинга / ПНЭО являются редкими опухолями и встречаются преимущественно у лиц молодого возраста, характеризуются высокой чувствительностью к ЛТ и ХТ. Лечение требует комплексного подхода и должно проводиться в специализированных центрах (рис. 3, 4).

\section{1. Локализованный опухолевый процесс}

\section{Общие принципы лечения}

При клинически локализованной форме заболевания к моменту установления диагноза 90\% больных имеют микрометастазы, поэтому локальное лечение (операция или ЛТ) должно обязательно дополняться системной (лекарственной) терапией. Комбинация ХT с локальными видами лечения (оперативным и/или лучевым) повышает 5-летнюю выживаемость с $10 \%$ до $60 \%$. После биопсии опухоли и тщательного обследования проводится 4-6 циклов индукционной ХТ в течение 12-24 нед., далее следует локальный метод лечения с последующим назначением от 6-10 циклов XT, проводящихся с интервалом 3 нед. Общая продолжительность лечения составляет 8-12 мес. Наиболее активными цитостатиками являются доксорубицин, циклофосфамид, ифосфамид, винкристин, дактиномицин и этопозид. Фактически все режимы лечения основаны на комбинациях из 4-6 перечисленных препаратов. Наиболее эффективные режимы лечения включают, как минимум, один алкилирующий химиопрепарат (ифосфамид или циклофосфамид) и доксорубицин. Добавление ифосфамида и этопозида в программу лечения значительно улучшило результаты терапии у пациентов с неметастатической Саркомой Юинга/ПнЭО.

\subsection{1. Хирургическое лечение}

Радикальная операция в тех случаях, когда она выполнима, расценивается как наилучшая возможность локального контроля. Хирургическая тактика лечения пациентов с саркомой Юинга кости и остеосаркомой не имеет принципиальных различий и проводится с соблюдением онкологических и ортопедических правил выполнения такого вида операций, описанных в предыдущем разделе. 


\subsection{2. Лучевая терапия}

ЛТ показана при невозможности радикального оперативного вмешательства и обсуждается в тех случаях, когда при гистологическом исследовании удалённого материала определяется недостаточный лечебный патоморфоз (то есть выявляется более $10 \%$ жизнеспособных опухолевых клеток). Нерадикальная операция с последующей лТ обладает сопоставимой эффективностью в сравнении с лТ в самостоятельном виде. Лт проводится в дозах 40-45 Гр при микроскопических остаточных опухолях и 50-60 Гр - при наличии макроскопических изменений. В качестве локального лечения при отказе от хирургического вмешательства или в случае нерадикальной операции применяется дистанционная 3D IMRT ЛТ в комбинации с XТ в РОД 2-2,5 Гр, СОД 60 Гр. При метастазах в лёгких - крупнопольная лТ в РОД 2 Гр, сОД 20 Гр.

\subsection{3. Химиотерапия}

Рекомендуемые режимы ХТ сарком семейства Юинга /ПНЭО представлены в табл. 3.

таблица 3. Рекомендуемые режимы химиотерапии сарком семейства Юинга/ПнЭО

Альтернирующий режим IE-VAC

IE: этопозид 100 мг/м² в/в в 1-5-й дни+ ифосфамид 1800 мг/м² в/в в 1-5-й дни (+ месна) каждые 3-4 нед.

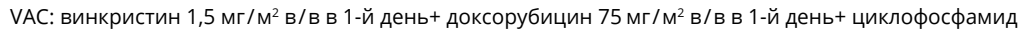
1200 мг/м² в/в в 1-й день, каждые 3-4 нед.

Винкристин 1,5 мг/м² в/в в 1-й день 1, 4, 7, 10, 13, 16, 19, 22, 25, 28, 31, 34, 37, 40 нед.

+доксорубицин 20 мг/м² в/в 4-часовая инфузия в 1-3-й дни 1, 7, 13, 19, 25, 31, 37 нед.

+ циклофосфамид 1200 мг/м² в/в в 1-й день 1, 4, 7, 10, 13, 16, 19, 22, 25, 28, 31, 34, 37, 40 нед.

+ дактиномицин 0,5 мг/м² в/в 4-часовая инфузия в 1-3-й дни 4, 10, 16, 22, 28, 34, 40 нед.

Винкристин 1,5 мг/м² в/в в 1-й день 1, 4, 7, 10, 13, 16, 19, 22, 25, 28, 31, 34, 37, 40 нед.

+ доксорубицин 20 мг/м² в/в 4-часовая инфузия в1 - 3-й дни 1, 7, 13, 19, 25, 31, 37 недели

+ ифосфамид 2000 мг/м² в/в (+ месна) в 1-й день 1, 4, 7, 10, 13, 16, 19, 22, 25, 28, 31, 34, 37, 40 нед.

+ дактиномицин 0,5 мг/м² в/в 4-часовая инфузия в 1-3-й дни 4, 10, 16, 22, 28, 34, 40 нед.

При раннем прогрессировании (в течение ближайшего года после комбинированного лечения)

Циклофосфамид 250 мг/м² в/в в 1-5-й дни+ топотекан 0,75 мг/м² в/в в 1-5-й дни, каждые 3-4 нед.

Этопозид 100 мг/м² в/в в1 - 5-й дни+ топотекан 0,75 мг/м² в/в в 1-5-й дни, каждые 3-4 нед.

Иринотекан 250 мг/м² в/в в 6-й день+ темозоломид 150 мг/м² внутрь или в/в в 1-5-й дни, каждые 4 нед.

Гемцитабин 900 мг/м² в/в 90-минутная инфузия в 1-й и 8-й дни+ доцетаксел 100 мг/м² в/в в 8-й день + Г-КСФ (филграстим 5 мкг/Кг п/К в 9-19-й дни)

\section{2. Метастатический и рецидивный опухолевый процесс}

Лечение метастатического процесса или местных рецидивов носит паллиативный характер за исключением больных с ограниченным рецидивом после длительного безрецидивного периода. Единственным прогностическим фактором, имеющим значение при рецидиве, является время его возникновения: длительность безрецидивного периода $\geq 2$ лет ассоциируется 
с улучшением отдалённых результатов лечения. Пациенты с метастатической формой заболевания должны получать такую же стандартную XT, как и больные с локализованным процессом с соответствующим локальным воздействием на метастазы (в основном с помощью Лт).

У больных с метастазами в лёгких повышение выживаемости может быть достигнуто путём резекции остаточных метастазов только после эффективной ХТ и тотального облучения лёгкого.

Метастазы в костях и костном мозге являются прогностически неблагоприятными (5-летняя выживаемость менее $20 \%$ ). При наличии метастазов в костях показана лт.

Применение интенсивных и высокодозных режимов ХТ с последующей аутологичной трансплантацией костного мозга оправдано у отдельных пациентов после тщательного обследования.

\section{4. НАБЛЮДЕНИЕ}

После комбинированного лечения больные саркомой Юинга должны наблюдаться с периодичностью 1 раз в 3 мес. в течение первых 2 лет, далее - 1 раз в 6 мес. до общей продолжительности 5 лет. Обязательными процедурами являются выяснение жалоб, осмотр, КТ грудной клетки, R-графия зоны установки эндопротеза, УзИ области послеоперационного рубца.

\section{ПЕРВИЧНОЕ

ОБСЛЕДОВАНИЕ ЛЕЧЕНИЕ \\ РЕСТАДИРОВАНИЕ}

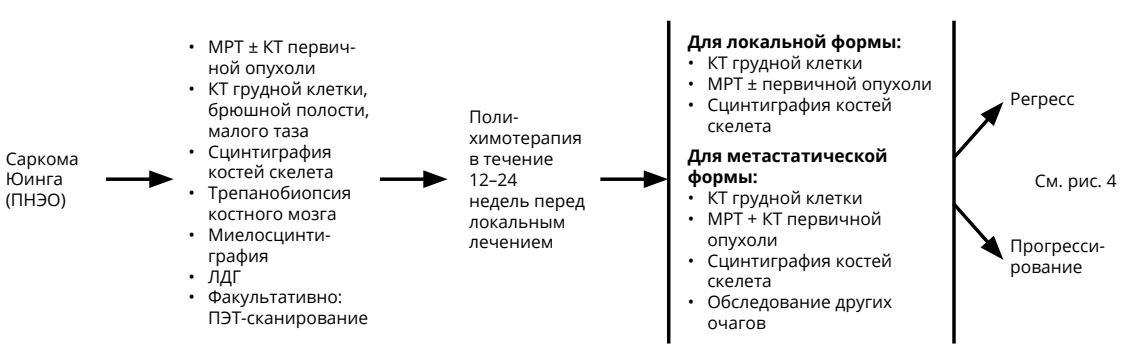

Химиотерапия включает комбинацию не менее трёх цитостатиков: ифосфамид и/или циклофосфамид, этопозид, доксорубицин, винкристин и Г-КСФ.

Рисунок 3. Рекомендуемый алгоритм диагностики и первичного лечения саркомы Юинга. 

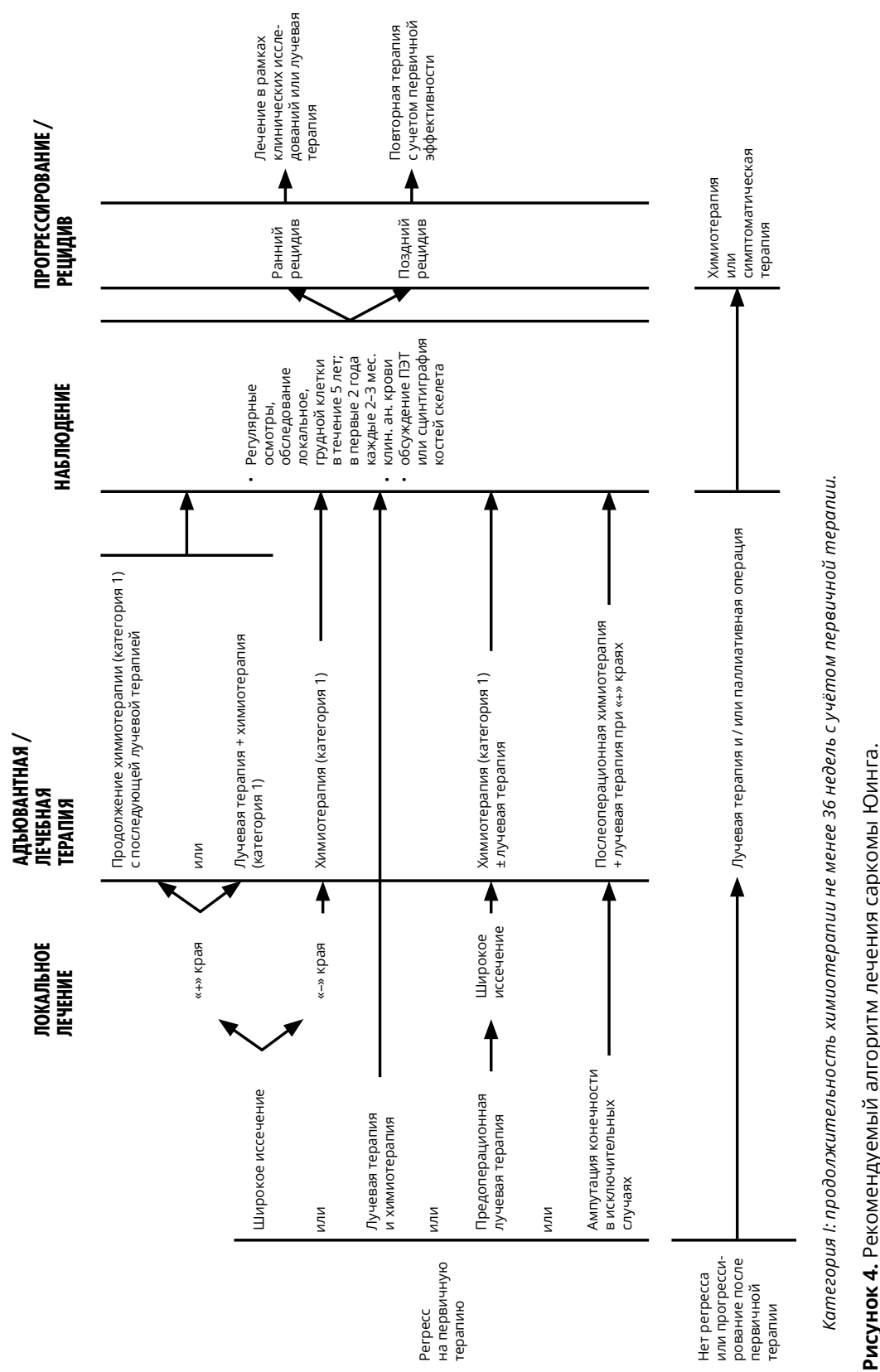